\title{
900,- Euro! oder Dreigroschen: Kollektive und individuelle Schaffensprozesse bei der Erarbeitung eines Theaterstückes
}

\author{
Michaela Reinhardt
}

\begin{abstract}
Zusammenfassung
Im vorliegenden Erfahrungsbericht werden drei Herangehensweisen vorgestellt, die zur Erarbeitung eines kleinen Theaterstückes mit Gruppen von italienischen Deutschstudierenden erprobt wurden. Dabei richtet sich das Augenmerk auf die Förderung kollektiver und individueller Schaffensprozesse innerhalb der Projektarbeit. Die angeführten Beispiele stammen aus der Arbeit im Rahmen des Projekts "TiLLiT" (teatro in lingua - lingua in teatro) der Universität Piemonte Orientale (Vercelli), welches seit zwölf Jahren Theaterarbeit in englischer, deutscher, französischer und spanischer Sprache fördert.
\end{abstract}

\section{Einleitung}

\subsection{Zum Theaterprojekt TiLLiT (teatro in lingua - lingua in teatro)}

Für alle Studierenden des Dipartimento di Studi Umanistici der Universität Piemonte Orientale (Vercelli /Italien) besteht die Möglichkeit, als Wahlfach einen Theaterkurs in Englisch, Deutsch, Französisch oder Spanisch zu belegen. Das Theaterprojekt TiLLiT (teatro in lingua - lingua in teatro) ${ }^{1}$ wurde vor zwölf Jahren ins Leben gerufen und ist seit 2006 integraler Bestandteil des Fremdsprachenstudiums. Für die Teilnahme erhalten die Studierenden 6 ECTS-Punkte. ${ }^{2}$ Entsprechend wird ihre Arbeit am Endes des Studienjahres nach festgelegten Kriterien beurteilt (s. Reinhardt 2010: 95 f.). Der zeitliche Rahmen von TiLLiT ist der Organisation des Universitätsbetriebes angepasst, d.h. die Veranstaltungen finden wöchentlich während des laufenden Semesters statt

\footnotetext{
${ }^{1}$ Das Projekt wurde 2004 von Marco Pustianaz und Michaela Reinhardt, Universität Piemonte Orientale, Vercelli entwickelt. Informationen: www.lett.unipmn.it/tillit.

2 Über die Kriterien der Leistungsbewertung werden die TeilnehmerInnen zu Beginn des Kurses in Kenntnis gesetzt. Dabei geht es selbstverständlich nicht in erster Linie um schauspielerische Fähigkeiten, sondern v.a. um den Einsatz, den die Studenten jeweils bezüglich der Arbeit in der Gruppe und ihrer individuellen Spracharbeit beweisen
} 
und haben die gleiche Stundenzahl wie die übrigen Kurse und Vorlesungen am Dipartimento (max. 2-4 Wstd.).

Studierende, die TiLLiT als Wahlfach belegen, müssen sich zu Beginn des Studienjahres verbindlich einschreiben. Am Ende des Wintersemesters nehmen sie an einem Theaterworkshop auf Italienisch teil (2x 4 Std.), der von einem ausgebildeten Schauspieler mit langjähriger pädagogischer Erfahrung durchgeführt wird. In diesen Stunden erhalten sie eine kleine Einführung in schauspielerische Techniken und lernen v.a., sich im Bühnenraum zu bewegen und $\mathrm{zu}$ artikulieren. Im Sommersemester findet die Probenarbeit in den einzelnen Sprachen statt (2 WStd.). Jede Gruppe erarbeitet unter der Leitung einer Lehrperson für die jeweilige Sprache einen kleinen Text zur Aufführung. Dabei kann es sich um bereits existierende Theaterstücke ${ }^{3}$ handeln, um Adaptionen oder um Texte, die erst in der Gruppenarbeit entwickelt werden. Am Ende des Sommersemesters führt jede Gruppe ihr ca. halbstündiges Stück im Rahmen eines Mini-Theaterfestivals auf. Außer der Teilnahme an den Proben werden die Studierenden dazu angehalten, nach Abschluss der Arbeit einen kleinen Erfahrungsbericht zu verfassen, in welchem sie die Theaterarbeit in ihren verschiedenen Aspekten sowie persönliche Erfahrungen und Lernprozesse reflektieren. ${ }^{4}$ TiLLiT hat darüber hinaus internationalen Charakter: Jedes Jahr werden nicht nur studentische Gruppen anderer italienischer Universitäten, sondern auch Gastgruppen aus dem Ausland eingeladen, die jeweils ein kleines Theaterstück in einer Fremdsprache aufführen.

\subsection{Konkreter Bezugsrahmen}

Der vorliegende Artikel bezieht sich speziell auf die Arbeit mit Gruppen von Deutschlernern („TiLLiT-Deutsch“). Diese bestehen durchschnittlich aus 7-12 TeilnehmerInnen, deren Sprachkenntnisse sehr unterschiedlich sind. In den letzten Jahren haben immer auch Nullanfänger teilgenommen. ${ }^{5}$ Die Tatsache, dass die Theaterarbeit für TeilnehmerInnen aller Niveaustufen möglich ist und sich bezüglich des Sprachniveaus immer eine heterogene Gruppe ergibt, hat sich - nach langjähriger Erfahrung - als uneingeschränkt positiv erwiesen. Studierende mit niedrigem Sprachniveau und sogar Nullanfänger können problemlos in die Arbeit einbezogen werden und profitieren von der Mitwirkung fortgeschrittener DeutschlernerInnen. Im Schutz des Spielrahmens (s.u., 2.1) fühlen sie sich nicht diskriminiert, sondern haben vielmehr aufgrund der multimodalen Art der Arbeit die Möglichkeit, ihre anfangs niedrige Sprachkompetenz auf anderen Ebenen der Schauspielarbeit zu kompensieren.

\footnotetext{
3 ,Theaterstück' wird hier und im Folgenden als Synonym für ,Theatertext' verwandt.

${ }^{4}$ Die Vorgaben zum Verfassen des Erfahrungsberichts sind insgesamt recht knapp: Die TN sollen auf max. fünf Seiten rückblickend den Verlauf der gesamten Arbeit reflektieren, wobei sie auf Aspekte der Spracharbeit, auf die Arbeit innerhalb der Gruppe und an ihrer individuellen Rolle eingehen. Dabei sollten sie positive sowie negative Erfahrungen und eventuell Verbesserungsvorschläge einbringen.

${ }^{5}$ Siehe z.B. Zusammensetzung der Gruppe TiLLiT-Deutsch 2015: 2 Nullanfänger, 1 TN mit Niveau A2, 1 TN mit Niveau B1, 2 TN mit Niveau B2, 1TN mit Niveau C1
} 
TeilnehmerInnen mit hohem Sprachniveau müssen selbstverständlich ihren Kenntnissen gemäß gefordert und gefördert werden.

\subsection{Ausgangspunkt: Förderung individueller und kollektiver Schaffensprozesse}

Bezüglich der Verbesserung sprachlicher Leistungen durch Theaterarbeit wurden inzwischen verschiedene Untersuchungen durchgeführt, und es liegen bereits positive Ergebnisse qualitativer empirischer Forschung vor (vgl. u.a. Reinhardt 2010, Passon 2011, Giebert 2012). In einer Umfrage ${ }^{6}$ im Rahmen des Projekts TiLLiT hat sich sogar gezeigt, dass die überwiegende Zahl der TeilnehmerInnen ${ }^{7}$ auch noch im Abstand von einem Jahr nach der Projektarbeit große Teile des eigenen Textes und des Textes ihrer MitspielerInnen erinnern und bestimmte Sätze und Strukturen in anderen Kontexten flexibel anzuwenden vermögen (Reinhardt 2010: 89 ff). Alle Befragten geben an, dass ihre Motivation die deutsche Sprache zu erlernen und speziell die Lust am Sprechen nach der Teilnahme am Theaterkurs stark zugenommen hat (Reinhardt 2010: 90). Aus den oben erwähnten Erfahrungsberichten wiederum geht besonders deutlich hervor, dass die Studierenden die Tatsache wertschätzen, persönlich an Entstehungsprozessen und an Regie-Entscheidungen beteiligt gewesen zu sein. Im gleichen Zusammenhang stellen sie zum einen die konstruktive Arbeit in der Gruppe als besonders förderlich für das Gelingen des Projekts heraus, zum anderen betonen sie den Stolz auf die Arbeit an der eigenen Rolle. Es scheint daher evident, dass sich die positive Erfahrung von Schaffensprozessen, kollektiven wie individuellen, direkt auf die Steigerung der Motivation und - was zu wünschen ist - indirekt auf die Verbesserung der Sprachkenntnisse auswirkt. Wenn dieser Aspekt offenbar eine so große Rolle spielt, sollte er bei der Planung der Arbeit seine besondere Berücksichtigung finden. In den vergangenen drei Jahren Theaterarbeit (2013 - 2015) habe ich dementsprechend versucht, möglichst viel Raum zur Förderung individueller und kollektiver Schaffensprozesse zu gewähren. Dies soll in der Skizzierung von drei Herangehensweisen zur Erarbeitung von Theaterstücken dargelegt werden. Im Kapitel 4 stehen die Ergebnisse einer kurzen schriftlichen Befragung, an der die Gruppen von 2014 und 2015 teilgenommen haben.

\section{Drei Herangehensweisen zur Erarbeitung kurzer Theaterstücke}

Bezüglich der Wahl oder Kreation des Theaterstückes gehe ich Jahr für Jahr unterschiedlich vor: Entweder präsentiere ich der Gruppe einen bereits fertigen Text, oder wir adaptieren ein Stück, oder wir erarbeiten gemeinsam etwas

\footnotetext{
${ }^{6}$ An der Umfrage nahmen insgesamt 30 Studierende aus den Gruppen TiLliT-Deutsch 2005, 2006 und 2010 teil.

${ }^{7}$ Im Folgenden unter der Sigla TN.
} 
völlig Neues auf Basis von Improvisation. Alle drei Vorgehensweisen lassen auf ihre Art Raum für die kreative Gestaltung der TN. Im Folgenden werden exemplarisch die letzten beiden Wege zur Erarbeitung eines Textes dargestellt, d. h. solche, bei denen nicht von einem fertigen Stück ausgegangen wird.

\subsection{Rahmenbedingungen}

Grundvoraussetzung für eine Theaterarbeit, die kollektive und individuelle Schaffensprozesse fördern soll, ist die gleichberechtigte Mitbestimmung der Studierenden in den diversen Phasen der Projektarbeit. Einzelziele und -aufgaben sollten während des Verlaufs immer wieder von der Gruppe ausgehandelt und definiert werden. Dabei sollte die Lehrperson vornehmlich eine übergeordnete Koordinatoren- bzw. Berater-Rolle einnehmen. Ihre Funktion ist es, Input zu geben, im Fall von Schwierigkeiten einzugreifen und Entscheidungen zu übernehmen. Darüber hinaus muss sie sprachliche Fehler korrigieren, entsprechende Übungen konzipieren, das Stück in seiner sprachlichen Gestaltung immer wieder überprüfen und eventuell ändern. Die Lehrperson behält also den Blick von außen, hält die Fäden zusammen und begleitet die Gruppe ermutigend bei ihrer Arbeit. Dennoch kann sie, je nach Bedarf, in bestimmten Momenten in eine Rolle schlüpfen, um Anregungen einzubringen, Ausspracheund Intonationsbeispiele zu liefern, den Fortlauf des Geschehens mitzugestalten, usw.. Die Funktion der Lehrperson im Wechsel von LiRo und LaRo-Technik ${ }^{8}$ wird ausführlich bei Manfred Schewe (2000: 96ff.) beschrieben.

Im Sinne der Förderung kooperativen Lernens (s. hierzu Johnson \& Johnson 1994; Johnson \& David W. 2009; Bonnet \& Küppers 2011) muss in bestimmten Arbeitsphasen der traditionelle universitäre/schulische Rahmen mit seinen Hierarchien aufgebrochen werden und ein Spielrahmen geschaffen werden, der das Experimentieren bzw. das spielerische Schaffen fördert. Das Konzept des Spielrahmens wurde von Ruth Huber mit Rekurs auf Erwin Goffmans frames entwickelt und erweist sich als äußerst gewinnbringend für die Drama- und Theaterpädagogik (vgl. hierzu Huber 2003: 381ff). ${ }^{9}$ Der Spielrahmen gewährt den TN Schutz und Sicherheit, ermuntert sie zu höchster Einsatzbereitschaft und sorgt gleichzeitig dafür, dass sich niemand überfordert fühlt (ibid.).

Ein weiteres Grundprinzip der Arbeit im Rahmen von TiLLiT-Deutsch ist, dass es keine Haupt- und Nebenrollen gibt, sondern alle TN gleichgewichtige Parts erhalten.

\subsection{Entwurf eines Theatertextes durch die Gruppe}

Beispiel 1: Ein Objekt als Ausgangspunkt -„Nur ein Spiel“ (TiLLiT-Deutsch 2013) Geht man von einem Objekt oder von einem Motiv aus und lässt die TN selbst in der Improvisation Szenen erfinden, gibt man ihnen die größtmögliche

\footnotetext{
8 LiRo steht für „LehrerIn in der Rolle“, LaRo für „LehrerIn außerhalb der Rolle“ (Schewe 2000: 96).

9 Zum Einrichten des Spielrahmens siehe auch Reinhardt 2006, 2010.
} 
Schaffensfreiheit in Bezug auf das Theaterstück. Dies erweist sich allerdings nur dann als sinnvoll, wenn solche Motive/Gegenstände genügend „echten“ Anreiz bieten und zum Spiel anregen. Die TiLLiT-Theatergruppe des Jahres $2013^{10}$ erfand auf der Basis des Motivs ,Apfel' verschiedene unterhaltsame Szenen, die sich zu einem kleinen Stück verbinden ließen. Die Szenen wurden beim ersten gemeinsamen Treffen in Kleingruppen in der Improvisation (in der Muttersprache) mit Äpfeln entwickelt und evozierten auf humorvolle Weise größere Themen wie Newtons Entdeckung der Schwerkraft, Wilhelm Tell und Schneewittchen sowie die Marktstrategien eines führenden Hardwarekonzern namens „Apfel“.

Schritt 1: Den TN wurden Äpfel verteilt und der Arbeitsauftrag gegeben, hiermit in drei Kleingruppen (zu dritt) jeweils eine kurze Szene zu entwickeln. Damit war der Spielrahmen abgesteckt. Die Improvisationen entsprangen zum Teil direkt aus der rein körperlich-sinnlichen Berührung mit den Äpfeln, des „Begreifens“ und Experimentierens. Hieraus und im gemeinsamen Spiel mit den Äpfeln ergaben sich Gesten und Bewegungen, die wiederum in Verbindung mit der Assoziation bekannter Motive zu Handlungen und kleinen Plots entwickelt wurden. Zum Beispiel wurde das In-der-Hand-Halten und Abwiegen zur Geste Schneewittchens, welches überlegt, ob es sich trotz seiner Apfelallergie erlauben kann, in die verlockende Frucht zu beißen (Kleingruppe 1). Auch die NewtonFigur tauchte spontan auf, aus der Geste des Werfens und Auffangens des Apfels, welche die Entdeckung der Schwerkraft suggerierte. Zu Schneewittchen gesellten sich die böse Königin und ein sprechender Spiegel. Die Kleingruppe 1 verband im Spiel den Märchenstoff mit der Newton-Geschichte und ließ Newton schließlich am vergifteten Apfel sterben.

Die Figur Wilhelm Tells dagegen entsprang aus dem spielerischen Umgang mit dem Apfel als einer Art Kultgegenstand (Kleingruppe 2). Tell wurde in die heutige Zeit projiziert, als Nutzer sozialer Netzwerke, der seine Selfies mit Apfel, Pfeil und Bogen auf Facebook postet und nebenbei das Leben seines Sohns auf das Spiel setzt. Als Zusatzfigur und running gag erfand die Gruppe Tells Frau, die sich immer ärgert, wenn ihr Mann ihr die Äpfel für den Strudel wegnimmt.

Eine weitere Gruppe von Figuren ergab sich aus dem Spiel mit dem Markennamen „Apfel“ und der neuesten Markterscheinung: einem Handy in Form eines Apfels, das sehr schnell große Missverständnisse auslöst (Kleingruppe 3).

Schritt 2: Die in der Improvisationsarbeit entwickelten Szenen wurden von mir ins Deutsche übertragen und beim folgenden Treffen gemeinsam gelesen und ausgefeilt. Einzelne Verse, die besonders schwierig auszusprechen waren oder Schlüsselmomente einer Szene darstellten, wurden mehrfach im Chor geübt (z.B.: „Spieglein, Spieglein an der Wand..."). Daraufhin wurde über die Rollenverteilung entschieden. In fast allen Fällen übernahmen die TN die gleichen Rollen, die sie von Anfang an in der Improvisationsarbeit eingenommen

\footnotetext{
${ }^{10}$ Die Gruppe bestand aus insgesamt neun TN, davon drei Nullanfängern, drei TN mit Niveau A2, drei TN mit Niveau B1
} 
hatten. Schließlich wurden die Szenen Schritt für Schritt gemeinsam geprobt. Da sie Eigenprodukte der TN waren, hatten diese ein besonderes Interesse, sie bestmöglich auszuarbeiten und in der Fremdsprache zu proben. Auch während der Arbeit am deutschen Text wurde weiterhin improvisiert und verändert.

Weiterer Verlauf: Im weiteren Verlauf der Proben wurden die einzelnen Szenen in einen choreographisch gestalteten Rahmen ${ }^{11}$ eingebunden und durch zwei weitere kleine Szenen ergänzt, welche die Studierenden später erfanden, so dass sich ein halbstündiges Theaterstück daraus ergab.

Kurzer Kommentar: Bei der oben beschriebenen Herangehensweise waren alle TN von vornherein gleich stark gefordert und gleichberechtigt mit ihren persönlichen Ideen in die kreative Arbeit einbezogen. Dies ermutigte sie, auch im weiteren Verlauf Regie-Entscheidungen zu treffen und sich gegenseitig Tipps zur Verbesserung zu geben. Entscheidungsschwierigkeiten gab es lediglich in Bezug auf den choreographischen Rahmen, da ein Teil der Gruppe zunächst fürchtete, den tänzerischen Ansprüchen nicht gewachsen zu sein. Den Übrigen gelang es aber, sie zu überzeugen und mit ihnen einfache und effektvolle Tanzschritte einzuüben, so dass am Ende alle TN mit ihrem Produkt zufrieden waren.

Die fremdsprachlichen Kompetenzen spielten in der ersten Arbeitsphase (Szenen-Kreation) noch keine Rolle. Bei der Konfrontation mit dem deutschen Text kam es darauf an, v.a. die Sprachanfänger nicht zu überfordern. Ihre Parts wurden größtenteils zunächst - in kleine Sequenzen zerlegt - solange im Chor gesprochen, bis sich die Anfänger sicher genug fühlten, sie allein auszusprechen. Den Fortgeschrittenen wurde selbstverständlich von vornherein entsprechend mehr abverlangt, was die sprachlichen Leistungen betrifft. Wichtig war, auch den deutschen Text jeweils eng an die in der Improvisation erwachsenen Gesten zu koppeln, um eine multimodale Sprachverarbeitung zu ermöglichen. Diese ist bekanntlich besonders effektiv im Bezug auf Gedächtnisleistungen, beim Erstwie beim Fremdsprachenerwerb (vgl. u.a. Huber 2003: 142ff.). Die Tatsache, dass es sich bei den zu lernenden Dialogen schlichtweg um die deutsche Übersetzung ihrer eigenen Textproduktionen handelte, schien die TN verstärkt zu motivieren, diesem Text auch in der Fremdsprache besonderen Ausdruck zu verleihen. In der Fremdsprachendidaktik werden solche Verfahren bekanntlich in Methoden wie dem Comunity-Language Learning bzw. des CounselingLearnings angewandt (vgl. Larsen-Freeman \& Anderson 2011: 85ff.), zu deren Grundprinzipien das Wahren der Selbstsicherheit der TN gehört.

Zu den nichtsprachlichen Lernzielen der oben beschriebenen Arbeit gehörten u.a. das Reflektieren über das Motiv ,Apfel' in Geschichte und Literatur ${ }^{12}$ sowie das Erwerben der Kompetenz, aus Versatzstücken ein in sich stimmiges Theaterstück zu entwickeln. Bei letzterem erwies sich die unterstützende und lenkende Hilfe der Lehrperson als unerlässlich.

Zufriedenheit und Stolz hinsichtlich der kollektiven Produktion kommen

\footnotetext{
${ }^{11}$ Die Musik wurde von den Studierenden gewählt, die Choreographie hingegen wurde von einzelnen TN entwickelt und mit der Gruppe eingeübt

12 Erstaunlicherweise wurde von den Studierenden nicht die Genesis zur Sprache gebracht
} 
exemplarisch in den folgenden Auszügen aus den Erfahrungsberichten von drei Beteiligten zum Ausdruck:

Wir hatten die Möglichkeit uns in unserer Arbeit frei zu fühlen. Die Lehrerin R. hätte uns auch einfach einen fertigen Text präsentieren können, ohne uns nach unserer Meinung zu fragen. In diesem Fall wäre es ein Kurs wie jeder andere gewesen. Aber dadurch, dass wir ein Produkt unserer eigenen Fantasie verwirklichen konnten, wurden die TiLLiT-Stunden zum großen Vergnügen. (Olga L., TiLLiT Deutsch 2013)

Beide Male waren es Kreationen der Gruppe, d.h. jeder hat seinen Beitrag an Ideen, Vorschlägen geliefert und die Aufführung war am Ende das Ergebnis der Gemeinschaftsarbeit. Die Stärke des Projekts besteht im Respekt vor dem Einzelnen als Individuum mit eigenen Ideen und Gefühlen, innerhalb einer Gruppe. (Alessio T., TiLLiT Español e TiLLiT Deutsch 2013)

Es war ein enormes Erfolgserlebnis etwas Neues zu schaffen, das bei der Aufführung großen Anklang fand. Und ich glaube, dass es etwas ist, was für lange Zeit in meiner Erinnerung und in der meiner Kommilitonen bleiben wird. (...) Ich kann sagen, dass ich mich jetzt Teil einer Gruppe fühle, mit der ich eine Erfahrung teile, die schwer im Leben zu wiederholen sein wird. (Eugenio A., TiLLiT Deutsch 2013)

\section{Ausgangspunkt Rahmenszene /Szenario}

Ein ähnliches Verfahren zwecks eigener Textproduktion besteht darin, eine Rahmenszene oder ein Szenario vorzugeben, welches von der Gruppe weiterentwickelt bzw. mit Inhalten und Figuren „gefüllt" wird. Wichtig ist, wie auch bei der oben beschriebenen Vorgehensweise, dass jede(r) TN für sich eine Rolle findet, die wirklich für sie/ihn reizvoll ist, und die zum Handeln herausfordert. Solche Rahmenszenen sollten darüber hinaus dem Erfahrungshintergrund der TN entspringen, um kreative Ausdrucksformen ihrer Lebenswirklichkeit zu ermöglichen (vgl. Poreda 2011:134). Szenarios, aus denen Theaterstücke entstanden, waren beispielsweise ein interaktiver Deutschkurs, bei dem die virtuellen Figuren ihr hinterhältiges Spiel mit den Anwendern treiben (TiLLiT-Deutsch 2007) und eine Therapie-Einrichtung zweier geschäftstüchtiger Damen, die ihren Patienten eine dubiose Methode namens „Stimmungstür“ verkaufen (TiLLiT 2010; vgl. hierzu Reinhardt 2010).

Beispiel 2: Entwicklung einer Handlung auf Basis einer Anfangsszene - „900,- Euro!“ (TiLLiT-Deutsch 2014) — Statt eines Rahmens oder Szenarios lässt sich auch mit einer Anfangsszene als Arbeitsvorschlag beginnen. Der Gruppe von $2014^{13}$ präsentierte ich folgende Szene in Skriptform, d.h. mit bereits fertig verfassten Dialogen:

Bei der Vernissage in einer Kunstgalerie verliert eine sehr reiche Dame ihr Portemonnaie. Als ein etwas trotteliger Aufseher dieses findet und

13 Die Gruppe bestand aus insgesamt sieben TN, davon zwei Nullanfängern, vier TN mit Niveau B1 und einer TN mit Niveau B2. 
vorübergehend auf einen Sockel legt, um das Geld daraus zu zählen, wird das Portemonnaie von Besuchern als „Kunst-Objekt" entdeckt. Die GalerieBesitzerin wittert sofort ein großes Geschäft, und der Aufseher wird zum Künstler deklariert.

Die Herausforderung, von dieser Szene ausgehend ein Stück zu erarbeiten, betrifft zunächst v.a.zwei Aspekte, mit denen sich die einzelnen TeilnehmerInnen direkt konfrontiert sehen: die Auseinandersetzung mit der Definition von,Kunst' und die Frage nach den handelnden Personen („Welche Figuren können für den Plot interessant sein, und welche Figur würde mich persönlich in diesem Zusammenhang reizen?"). Daran schließt sich die Frage nach dem möglichen Handlungsverlauf an.

Schritt 1: Bevor die TN mit der Szene konfrontiert wurden, fanden Improvisations- und Aussprachespiele als einführende Arbeitsschritte statt. Bei der ersten Übung stellte ich einen aufgespannten Regenschirm in die Mitte des Raumes und erklärte ihn zum Kunstwerk. Die TN reagierten etwas ratlos und verlegen. Daraufhin bat ich die Gruppe, sich in die Rolle gelangweilter Teenager zu versetzen, die von ihrer Kunstlehrerin (diese Rolle übernahm ich) in eine Ausstellung geführt werden, um dieses Kunstwerk zu betrachten. ${ }^{14}$ In diesem Spielrahmen entstand sofort eine entspannte Atmosphäre mit Grüppchen junger Leute, die hinter meinem Rücken tuschelten, sich Zeichen gaben und Fratzen schnitten, während ich das Kunstwerk „Regenschirm“ erklärte. Anschließend sollte sich die Gruppe in die Rolle von Personen versetzen, die sich als Kunstkenner ausgeben und kluge Reden schwingen, und schließlich in einem dritten Übungsschritt in Galeriebesitzer, die ihrer Klientel das Werk verkaufen wollen.

Eine weitere Übungs-Sequenz, die dem Lesen der Anfangsszene vorgeschaltet wurde, bestand darin, Definitionen für Kunst zu finden. Daraufhin wurde zunächst die Frage (auf Deutsch) „Was ist Kunst?“ laut geübt, dann leise, in verschiedenen Tonarten und Stilen (nach Queneau 2007) ${ }^{15}$, im Chor und nacheinander gesprochen, bis sich eine Art Klangteppich im Raum ergab. Danach sollten die TN knappe Definitionen laut sprechen: „Kunst ist Phantasie“ - „Kunst ist Magie!“ - „Kunst ist Provokation“- „Kunst ist Faszination“- Kunst ist Chaos und Anarchie“- „Kunst ist ein Skandal!“- „Kunst ist ein Missverständnis“„Kunst ist Inszenierung“. Wieder wurden die Sätze zunächst einzeln, mal laut, mal leise oder geflüstert, in unterschiedlichen, ihnen entsprechenden Tonarten ausgesprochen und im Chor geübt. Jede(r) TN suchte sich schließlich einen Satz aus und versuchte ihm eine adäquate Klanggestalt zu verleihen. Am Ende wurde ein Chor erstellt, in dem sich die Sätze und Stimmen überlagerten, an-

\footnotetext{
14 Diese Übung fand in der Muttersprache statt, nicht nur, um auch die Nullanfänger der Gruppe gleichberechtigt zu beteiligen, sondern auch, weil es hier vornehmlich darum ging, Einstellungen und Haltungen zu erproben

15 Von Queneau wurde hier die Idee übernommen, eine Äußerung in unterschiedliche Situationen /Kontexte zu setzen und entsprechend in unterschiedlichen Stilen vorzutragen. Während Queneau in seinen „Stilübungen“ Text und Sprachregister jeweils ändert, bleiben bei der hier angewandten Übung die Äußerungen wörtlich unverändert. Die Stilvariationen finden vielmehr in Klang, Ton und Lautstärke ihren Ausdruck.
} 
und abschwollen und zu einem beeindruckenden Klangerlebnis wurden. Die Gruppe beschloss, ihre kleine Klangkomposition in das zu erarbeitende Stück einzubinden.

Schritt 2: Nach diesen beiden Übungsphasen wurde der Szenenvorschlag (s.o.) gemeinsam gelesen. Die Studierenden nahmen ihn an und beschlossen, bis zum nächsten Treffen zu überlegen, welche Rolle sie jeweils innerhalb des vorgegebene Szenarios einnehmen könnten. Darüber hinaus bekamen sie den Auftrag, Definitionen zu Kunst sammeln.

Schritt 3: Beim darauf folgenden Treffen wurde in der Mitte des Raumes ein Portemonnaie ausgestellt. Die TN sollten sich nun in unterschiedliche Rollen ihrer Wahl versetzen und verschiedene Reaktionen ausprobieren, die ihre jeweilige Figur in der Konfrontation mit dem ,Kunstwerk' zeigte. Dabei konnten auch Rollen der bereits vorgegebenen Anfangsszene gewählt werden. Relativ schnell entstand ein buntes Panorama von Figuren. Zu den bereits vorhandenen gesellten sich die Figur einer bizarren russischen Künstlerin als Gegenspielerin zum „Pseudo-Künstler“ (Galerie-Aufsicht) und ein weiterer Aufseher der Galerie - ebenfalls als Gegenspieler zum ersten Aufseher. Es trat außerdem ein kleines freches Mädchen hervor, das gelangweilt mit seinen Eltern durch die Galerie ging und das Portemonnaie als „nur ein Portemonnaie!“ entlarvte.

Im Anschluss an die Probe wurden Definitionen von Kunst ausgetauscht und entsprechende Sätze zu den bereits in der ersten Stunde verwandten Definitionen ergänzt. (z.B. „Kunst ist Freiheit“ - Imagination!“ - „Kunst ist Kapital!")

Weiterer Verlauf: Schritt für Schritt wurden Anschluss-Szenen improvisiert oder gemeinsam im Gruppengespräch ausgearbeitet und anschließend von mir ins Deutsche übertragen. Beim Fortschreiben der Handlung ergaben sich weitere Figuren: ein Fernsehreporter mit Kamerateam, eine Kunstexpertin und ein Auktionator. Der Plot entwickelte sich dahin, dass die reiche Dame, die ihr Portemonnaie mit 900,- Euro verloren hatte, es schließlich als ,Kunstwerk' für 900.000 Euro ersteigert. Am Endes des Theaterstückes steht der Klangteppich aus Kunst-Definitionen, von den einzelnen Figuren, in einer Reihe zum Publikum gerichtet, vortragen.

Kurzer Kommentar: Auch bei dieser Herangehensweise stehen in der Anfangsphase nicht die fremdsprachlichen Fähigkeiten im Vordergrund, sondern ein Spielangebot in der Muttersprache. Während die TN in Gruppen größtenteils stereotypen Rollen und bestimmte Haltungen erproben und darzustellen versuchen, brauchen sie sich zunächst auch nicht individuell zu exponieren. Gleichzeitig werden sie einzeln herausgefordert, sich mit der Definition von Kunst auseinanderzusetzen und anschließend der Gruppe eigene Vorschläge zu unterbreiten. Die darauf folgenden Aussprache-Übungen auf Deutsch finden ebenfalls im Schutz der Gruppe bzw. des Spielrahmens statt („Was ist Kunst?“), so dass jede(r) TN zunächst Zeit hat, genügend Sicherheit zu gewinnen. Im dritten Schritt sind die TN explizit in ihrer individuellen Kreativität gefordert, indem sie eine Figur aus sich heraustreten lassen, die auf das vermeintliche Kunstwerk reagiert. Sie haben dabei größte Wahlfreiheit und können die 
eigene Figur im Laufe der Arbeit kontinuierlich weiter ausgestalten. Dies geschieht in der Interaktion mit den anderen (s.u., 3.2) und im Prozess des gemeinsamen Erarbeitens des Handlungsverlaufes. Dabei werden nach und nach die fremdsprachlichen Ausdrücke und Strukturen (wie z.B.: „Ich merke, Sie verstehen was davon.“- „Was für ein Quatsch!“- „Guck mal, das ist doch nur ein Portemonnaie!" usw.) an die Handlungen dieser Figur geknüpft. Kollektive und individuelle Schaffensprozesse sind bei dieser Arbeit eng miteinander verzahnt, wie u.a. auch im folgenden Ausschnitt des Erfahrungsberichts einer Beteiligten deutlich wird:

Es war schön, von einer Basis-Idee [...] auszugehen und diese auf unterschiedliche Weise, individuell und kollektiv, weiterzuentwickeln, bis hin zum Einklang unserer Ideen in einer Richtung, die zum definitiven Text führte, über den langen Weg eines intensiven und ständig fortlaufenden Erarbeitungsprozesses. (Barbara M., TiLLiT Deutsch 2014)

Beispiel 3: Adaption eines Klassikers -„Für drei Groschen“ (TiLLiT-Deutsch 2015) - Die dritte Möglichkeit, die hier beschrieben werden soll, besteht in der Bearbeitung eines bereits vorgegebenen Textes. In diesem Fall scheint auf den ersten Blick die Schaffensfreiheit der TN eingeschränkter zu sein als im Vergleich zu den oben genannten Fällen. Doch kann auch hier gerade die Begeisterung für den Text, wie im Beispiel der „Dreigroschenoper“ von Bertolt Brecht (Theatergruppe $2015^{16}$ - der Vorschlag kam von den TN selbst -), Auslöser für sehr kreative und anregende Gruppenarbeit sein. Kürzungen und Änderungen des Textes sind innerhalb der Gruppe auszuhandeln. Darüber hinaus ist bei dieser Herangehensweise die freie Wahl und Ausgestaltung der Rollen (s.u., 3.2) besonders wichtig.

Schritt 1: Da diesmal von einem bereits vorhandenen Text ausgegangen wurde, musste zunächst sichergestellt werden, dass alle TN diesen gut kennen. Die Hälfte der Gruppe hatte die Dreigroschenoper im Vorjahr im Literaturunterricht ausgiebig behandelt. Den übrigen Studierenden wurde empfohlen, sich den Text zu beschaffen und zu lesen (die Nullanfänger lasen ihn in der italienischen Übersetzung). Den Proben vorangestellt wurde ein gemeinsames Gespräch über dieses Theaterstück, über das Werk Brechts und dessen wichtige Stellung in der Theatergeschichte. Auch während der Probenarbeit wurden immer wieder kurze Gesprächseinheiten eingeschoben, um sich den Originaltext der Dreigroschenoper und Brechts Intentionen vor Augen zu halten.

Es wurde beschlossen, sich des Kunstgriffs des „Theaters im Theater“ zu bedienen, um die Gruppe von dem Leistungsdruck zu befreien, den hohen Ansprüchen bezüglich eines Brecht'schen Stückes gerecht werden zu müssen. Denn eine Schauspieltruppe darzustellen, die Brechts Theatertext probt, bedeutet, nicht perfekt sein zu müssen. Im Gegenteil: Gerade die Unfähigkeit,

\footnotetext{
${ }^{16}$ Die Gruppe bestand aus insgesamt sieben TN, davon zwei Nullanfängerinnen, zwei TN mit Niveau B1, zwei TN mit Niveau B2 und einer TN mit Niveau C1
} 
Brechts Text und Konzepte überzeugend darzustellen, reizte die Gruppe als mögliches Leitmotiv für ihr Stück. Damit war die Richtung der Arbeit am Text vorgegeben. Das Stück wurde inhaltlich wiefolgt konzipiert: Eine Theatergruppe probt die Dreigroschenoper von Brecht. Die ehrgeizige, aber wenig kompetente Regisseurin zeigt bei der Arbeit wenig Sinn für Brecht'sche Maximen. Gleich zu Anfang nimmt sie einer Schauspielerin ihre Rolle und besetzt diese mit ihrer eigenen Tochter, welche sich als äußert schlechte Schauspielerin erweist und somit die Arbeit der übrigen Truppe kompromittiert. Darüber hinaus kassiert die Regisseurin Gelder von einem dubiosen Sponsor, während die Truppe vergeblich auf Bezahlung warten muss. Die Schauspieler planen schließlich, sich an Mutter und Tochter zu rächen.

Schritt 2: Als Einstieg machte die Gruppe Gangübungen, die jeweils den drei bei Brecht vorkommenden gesellschaftlichen Gruppen entsprachen: zunächst bewegten sich alle TN als „Bettler“ im Raum, danach als „Banditen“ und schließlich als „Prostituierte“. Diese Gangübungen wurden zur Basis für die Auftritte der jeweiligen Gruppen im Stück, in welchem alle TN - je nach Szene - als Banditen, Bettler oder Prostituierte agierten.

Schritt 3: Nachdem man beschlossen hatte, welche Szenen aus dem Original übernommen werden sollten, führte die Gruppe zahlreiche Ausspracheübungen kurzer Dialog-Abschnitte dieser Szenen im Chor und mit jeweils wechselnder Besetzung durch. Zunächst wurde an zwei Textstellen solange gefeilt, bis regelrechte „Mini-Aussprache-Produkte“entstanden. Als erstes wurde folgender Ausschnitt abschnittsweise auf jeweils 2 TN verteilt und anschließend mit der Hilfe von Stilübungen (nach Queneau, s.o.) geprobt: „Tief im Herzen Sohos/ feiert der Bandit Mackie Messer /seine Hochzeit mit Polly Peachum,/ der Tochter des Bettlerkönigs.“ (Brecht 1968: 17). Dabei sollten die TN-Paare ihren individuellen Textabschnitt jeweils in Stil und Ton selbst gestalten. Im zweiten Abschnitt ging es darum, einer Replik der Lucy-Figur besonderen Ausdruck zu verleihen: „Du gemeiner Schuft! Wie kannst du mir noch ins Gesicht sehen!!“ (ibid. 60). Auch dieser Abschnitt wurde im Chor und mit wechselnder Rollenbesetzung (im Dialog mit Mackie Messer: „Aber Lucy, hast du denn gar kein Herz?") und in verschiedenen Nuancen geprobt, bis alle TN ihn auswendig spielen konnten. Ähnlich wurde nach und nach mit vielen weiteren Szenenabschnitten verfahren, bis alle TN einmal die Möglichkeit hatten, die Stimmen aller Figuren des Stückes zu erproben.

Schritt 4: Erst nach ein paar Wochen gemeinsamer Arbeit wurde die Frage nach der Rollenverteilung gestellt. Jede(r) TN sollte diesbezüglich eine erste und zweite Wahl treffen. In den meisten Fällen hatten sich die TN inzwischen jeweils mit einem bestimmten Part angefreundet, bzw. es hatte sich herausgestellt, wer welche Rolle besonders überzeugend spielte. Nur in einem Fall gab es Überschneidungen bei der Rollenwahl. Diese ließen sich aber im Gruppengespräch schnell aufheben, und die Teilnehmerin, die auf ihre zweite Wahl ausweichen musste, eignete sich im Laufe der Proben ihren Part erfolgreich an.

Weiterer Verlauf: Zwecks bestmöglicher Gestaltung der individuellen Rollen 
wurden - diesmal jeweils in der Rolle - abermals Gangübungen durchgeführt, imaginäre „Gruppenfotos" erstellt und einzelne Dialog-Abschnitte geprobt. Es ging hierbei darum, Fragen zu klären, wie z.B.: Wie bewegt sich der autoritäre Bettlerkönig Jonathan Peachum? Wie reagiert Celia Peachum, als sie von der heimlichen Hochzeit ihrer Tochter erfährt? Und wie verhält sie sich, als die Huren das versprochene Geld von ihr einfordern? Wie und in welchem Ton redet Mackie Messer mit seinem Freund, dem Sheriff von London?

Nicht immer leicht war es, die doppelte Funktion bestimmter Figuren klar herauszuarbeiten: die der Schauspieler in der Rolle einer Rolle. Dieses und ähnliche Probleme wurden in der Gruppe diskutiert, und alle TN fühlten sich ermuntert, Tipps und Ideen einzubringen. Daraus ergab sich ein konstruktives und stimulierendes gemeinsames Regie-Führen, wie auch der folgende Auszug aus dem Erfahrungsbericht eines TNs belegt:

Einige Entscheidungen haben eine offene und tiefgehende Diskussion unter uns ausgelöst, und jeder hatte das Vergnügen alle eigenen Überlegungen oder Einstellungen zu den Fragen mit den Kommilitonen zu teilen, alles im Zeichen des gegenseitigen Respekts. Wir haben unter uns Teilnehmern unser schöpferisches Potenzial mit Einsatzbereitschaft und Ernsthaftigkeit zum Ausdruck gebracht, uns den anderen gegenüber in einer Auseinandersetzung geöffnet, die von Verständnis und Neugier geprägt war. (Riccardo B., TiLLiT Deutsch 2015)

Kurzer Kommentar: Bei dieser Herangehensweise kommt die kreative Arbeit der Gruppe und der Einzelnen v.a. in zwei Bereichen zum Tragen: zum einen bei der gemeinsamen Neubearbeitung des Textes, zum anderen in der individuellen Gestaltung der Figuren. Bei der Bearbeitung eines literarischen Kunstwerkes wie der Dreigroschenoper muss darauf geachtet werden, dass entsprechende Leerstellen im Text ausgemacht werden, die sich füllen lassen. Dem rezeptionsästhetischen Ansatz entsprechend (für den FSU: vgl. Mummert 2006) verstehen sich die TN auch im Falle einer literarischen Textvorlage als MitgestalterInnen / Bedeutungsschaffende. Insofern ergibt sich automatisch eine fruchtbringende Auseinandersetzung mit Werk und Autor. Dabei kann die Entdeckung der eigenen künstlerischen Fähigkeiten als positiver überraschender Nebeneffekt wiederum stimulierend wirken. So erwies sich auch das Erfassen des künstlerischen Wertes von Brechts Text und dessen ästhetischen Zeichencodes (Reinhardt 2014) als wichtiger motivationaler Faktor. Gerade beim Arbeiten mit vorgegebenen Texten ist die Theaterarbeit immer auch Zugang zum Stück selbst: fachdidaktisch gesehen im Rahmen der handlungs- und produktionsorientierten Methoden als Zugang zu literarischen Werken - bekanntlich ein Ansatz, der in den 1990er Jahren besonders populär wurde und auf Schaffensprozesse aufbaut. Dass im Fall von „Für drei Groschen“ der neue Text von den TN als ihr eigenes Produkt gesehen wurde, kommt sehr deutlich bei den Ergebnissen einer kurzen Befragung (s.u., Kap. 4, insb. Zitat Riccardo B.) zum Ausdruck.

Der zweite Bereich, das Gestalten der individuellen Rolle, soll weiter unten im Rahmen der allgemeinen Überlegungen (Kapitel 3.2) etwas ausführlicher behandelt werden. 


\section{Weitere allgemeine Überlegungen}

Im vorliegenden Kapitel werden drei übergeordnete Aspekte angesprochen, die für alle oben beschriebenen Herangehensweisen von gleicher Bedeutung sind.

\subsection{Lernen als archetypischer individueller Schaffensprozess}

Für alle an der Theaterarbeit Beteiligten, und im besonderen Maße für die Nullanfänger in der Gruppe, bedeutet das Lernen ihres Textes das direkte Koppeln fremdsprachlicher Ausdrücke und Strukturen an Handlung und Gesten, d.h. die Codierung des neuen Sprachmaterials geschieht im Zusammenspiel mit körperlicher Geste und mit Emotionen. Dieses Konstruieren einer eigenen Erfahrungswelt in der Fremdsprache erscheint als besonders wirksam für das Erinnern sprachlicher Strukturen. Nach Huber schaffen sich LernerInnen ihre Sprachwelt aufgrund essenzieller, sensomotorischer und emotionaler Erfahrungen, bzw. in der direkten Koppelung an solche Erfahrungen. Sprachenlernen - ob Muttersprache oder Fremdsprache - erweist sich demnach bereits als archetypischer schöpferischer Vorgang (Huber 2003: 152), welcher an alle weiteren Schaffensprozesse gekoppelt ist. Daher sollte multimodale Verarbeitung, wie sie die Theaterarbeit ermöglicht, in besonderem Maße gefördert werden.

\subsection{Das Gestalten der eigenen Rolle}

Um die Schaffensfreude der TN an ihren Figuren so gut wie möglich zu fördern, müssen diese im Stück als Ausdruck eines „Möglichen Selbst“ aus den TN heraustreten bzw. in der Interaktion entstehen können (Huber 2003: 431ff), d.h. eigene Kreation der TN sein. Ein „Mögliches Selbst“ kann z.B. das „Angst-Ich“ oder das „Wunsch-Ich“ eines Individuums sein (ibid. 433). Wie weiter unten (Kap. 4) deutlich wird, wählt die Hälfte der TN gern Rollen, die sie als weit entfernt von ihrem Alltags-Selbst empfinden, als eine Art eigene „innerer Gegenspieler" (Max Frisch, zitiert ibid.).

Das Hervortreten der Figuren geschieht am besten in spielerischen Situationen, die zum spontanen Handeln herausfordern. Geht man von einem fertigen Text und von vorgegebenen Figuren aus, müssen diese „entdeckt“ und erspürt werden können. Die TN müssen ihre Figur frei wählen und selbst gestalten dürfen. Die Annäherung an eine literarische Figur kann auf verschiedene Weise geschehen: über das Sprechen, durch Gangübungen oder im Zusammenspiel mit anderen Figuren (s.o., 2.3).

Dass bei der oben beschriebenen Theaterarbeit das Gestalten der eigenen Rolle als individueller Schaffensprozess im Kontext des Kollektiven empfunden wurde, zeigt exemplarisch der folgende Ausschnitt. Er stammt aus dem Erfahrungsbericht der Teilnehmerin des Dreigroschen-Projekts, welche die „schlechte Schauspielerin“ (Susanne) darstellte und in der Rolle von Polly 2 der Theatertruppe die Inszenierung zu verderben drohte. 
Sich auf das Spiel einzulassen, bedeutet auch über sich selbst lachen zu können. Und meine Figur (Susanne-Polly 2) hat mich genau dies gelehrt. Indem ich mich nicht allzu ernst genommen habe, habe ich mich in die Figur eingefühlt und versucht das Beste herauszuholen, um in einer Sprache aus Gesten und Worten dem Publikum zu verstehen zu geben, wer Susanne-Polly2 ist. Es war nicht ich, Elisabetta, die auf der Bühne handelte und sprach, sondern Susanne-Polly2. All dies war nur möglich dank des aktiven und anteilnehmenden Zuhörens und der Ratschläge und Ideen seitens der Personen, die um mich herum waren, und schließlich wiederum durch meine eigenen Vorschläge und Ideen zur Verbesserung der Figur Susanne in ihrem Auftreten und Handeln. (Elisabetta C., TiLLiT Deutsch 2015)

Im diesem Zitat kommen zwei wichtige Aspekte zum Tragen: Eine neue Rolle einnehmen heißt auch (humorvoll) Abstand zu sich selbst gewinnen können. Darüber hinaus ist auch bezüglich der Rollenfindung der Aspekt des Kollektiven ausschlaggebend (Wie sehen die anderen meine Figur? Was erwarten sie von ihr?). Alle Figuren entstehen auch im Austausch mit den anderen TeilnehmerInnen (s. hierzu auch Franz \& Hesse 2011: 109f.). Es erwächst so ein neues internes soziales Geflecht, das TN und Figuren gleichsam mit einschließt und bei dem das solidarische Mitgestalten der Figur durch die Anderen oder ihre begeisterte Anerkennung entscheidend auch zur Verbesserung des individuellen Selbstkonzepts ${ }^{17}$ und zu einer konstruktiven Persönlichkeitsbildung beitragen kann. ${ }^{18}$

Huber bezeichnet das Gestalten der eigenen Figur als „das magische Als-ob des Fremdsprachlers" (ibid. 437ff.). Sie weist darauf hin, dass fremdsprachige Figuren oft sogar ausdrucksvoller sind als muttersprachliche und erklärt dies folgendermaßen:

Zum einen hat die Theaterfigur gemessen am Schülerstatus so etwas wie eine soziale und identitäre Karriere gemacht, ihre Selbstsicherheit bringt dies zum Ausdruck: als Studentin war ich nur eine Nummer, als Person in einem Theaterstück bin ich wer und habe etwas zu sagen! (Huber 2003: 437)

Beim Identisch-Werden mit der Figur kann es zu einem Flow-Erlebnis kommen, welches die Motivation steigert, die Lust am Lernen/ Schaffen erhöht und somit ein gutes Gelingen und Behalten der fremdsprachlichen Produktion ermöglicht. ${ }^{19}$ Nach Huber wird das resignierte „ich kann nicht“ des Lerners übertrumpft im Kunstgriff des „Ich tue so, als ob ich könnte“. Dies lässt sich in folgender „Zauberformel“ zusammenfassen: „Ich handle, als ob ich

\footnotetext{
17 Zum Begriff des Selbstkonzepts siehe. u.a. Huber 2003: 436.

${ }^{18}$ In den Erfahrungsberichten der Studierenden spiegelt sich auch dieser Aspekt wieder

${ }^{19}$ Auch in Bezug auf das Erlernen von Wissenschaftssprache ist Theaterarbeit hilfreich, insofern es das Sich-in-eine-Rolle-Begeben ermöglicht und damit eine Habitus-Aneignung: „Die Verinnerlichung wissenschaftssprachlicher Strukturen erfolgt über das Sich-zu-eigen-Machen von vorgegebenen Sätzen (in einer Rolle, Anm. MR) und die schöpferische Auseinandersetzung mit diesen" (Reinhardt 2009: 311).
} 
Schauspieler wäre, ich gebärde mich, als ob ich diese Person wäre und ich rede, als ob ich Deutsch könnte" (Huber 2003: 440).

\subsection{Motivationssteigerung durch kleine Zwischen-Produkte (Nahziele)}

In der Sprachlehrforschung wird heute Motivation generell als dynamisches Konstrukt gesehen, welches das Individuum in den Mittelpunkt stellt (Kleppin 2004: 4). Stärker als früher werden kognitive und affektive Komponenten sowie die Bedingungsfaktoren des FSUs in Überlegungen zur Motivationssteigerung miteinbezogen (ibid. 4f). Kognitive und affektive Komponenten fallen bei jedem Individuum anders aus und umfassen Ziele und Erwartungen, Kontrollüberzeugung, Selbstkonzepte, Anstrengung, Ängstlichkeit, Hilflosigkeit, Freude an der Tätigkeit selbst usw. (ibid.). Als wichtiger Aspekt wird in der Forschung hervorgehoben, dass Lernende entweder sich selbst oder einer äußeren Instanz den Erfolg bzw. Misserfolg von Lernprozessen zuschreiben. „Motivierend scheint ein Erfolg besonders dann zu sein, wenn der Lernende dessen Ursachen im eigenen intentionalen Arbeitseinsatz begründet sieht", stellt Claudia Riemer fest (Riemer 2004: 40, zitiert nach Hoffmann 2008: 99). Eine bedeutende Rolle für die Motivationssteigerung spielen überdies kurzfristige Erfolge. So erscheint es besonders wichtig, Nahziele zu setzen:

$\mathrm{Zu}$ beobachten war in Beratungssituationen, dass Lerner - wenn sie sich auf konkrete Nahziele konzentrieren, die dann auch in einer späteren Beratungssitzung evaluiert wurden, Zufriedenheit mit dieser Lösung äußerten und ihre Anstrengungen weiter fortführen wollten (Kleppin 2004: $5)$.

Bei der Theaterarbeit sollten den TN demnach regelmäßig konkrete Nahziele geboten werden, die sie zum Weiter-Schaffen ermutigen. Dies betrifft alle Ebenen der Arbeit. Nicht nur für Nullanfänger kann beispielsweise die Aussprache eine große Hürde bedeuten. Daher sollten von Anfang an kurze Dialoge, Satzabschnitte oder auch nur bestimmte Ausdrücke in Chor-Übungen - möglichst spielerisch - geprobt werden (s.o). Besonders ratsam ist es, hierbei Schlüsselmomente bzw. Schlüsselbegriffe im Text zu wählen, um von vornherein Sprache auch in ihrem Bedeutungszusammenhang herauszuarbeiten. Diese kurzen Ausschnitte sollten in der Anfangsphase der Proben jedes Mal erneut aufgenommen und perfektioniert werden, so dass die TN die eigenen Fortschritte wahrnehmen und die gelungenen Dialogabschnitte als kleine Produkte ihrer Arbeit erfahren können.

Ebenfalls wirksam bezüglich der Motivationssteigerung ist das Proben und Perfektionieren kurzer Szenenausschnitte. Hier geht es außer um den Text vor allem um die szenische Umsetzung. Wenn eine Mini-Szene einmal „steht“ und allgemeine Zufriedenheit auslöst, ist es erfahrungsgemäß leicht im Flow zu bleiben. Die Lust zum Weiter-Schaffen steigert sich automatisch, wenn solche Szenen gefilmt und als erste kleine Kreationen über digitale Medien ausgetauscht werden können. 
Klaus Holzkamp bringt bereits 1983 den kollektiven Aspekt von Motivation ins Spiel - er spricht in seiner "Grundlegung der Psychologie“ von „sozialer Motivation":

[als] Motivation wird mithin nicht nur der Erfolg der eigenen Aktivität [...] antizipiert, der Erfolg der individuellen Aktivität ist vielmehr daran gemessen, wieweit sie zum Gesamterfolg des jeweiligen kollektiven Unternehmens beiträgt (Holzkamp 1983: 170, zitiert nach Hoffmann 2008: 102).

\section{Ergebnisse einer schriftlichen Befragung}

Um der Frage nach dem positiven Einfluss der unterschiedlichen Schaffensprozesse weiter nachzugehen, habe ich im Jahr 2015 erstmals nach Abschluss der Arbeit und der Abgabe der freien Erfahrungsberichte einen kurzen Fragebogen (s. deutsche Übers. im Anhang) ausfüllen lassen. Dieser Fragebogen bestand zum größten Teil aus geschlossenen Auswahl- und Listenfragen und halboffenen Fragen. Insgesamt gab es 10 Befragte aus den beiden Gruppen von 2014 und 2015 (,900,- Euro!“ und „Für drei Groschen“). Die wichtigsten Ergebnisse werden im Folgenden kurz zusammengefasst.

1. Alle TN fühlen sich motivierter als vorher (die große Mehrheit sogar viel motivierter als vorher), die deutsche Sprache weiter zu lernen und geben an, ihres Ermessens Fortschritte in den folgenden Bereichen gemacht zu haben:

- Aussprache: 10/10 große Fortschritte;

- Sprachverstehen: 7/10 große Fortschritte, 3 mäßige Fortschritte;

- Satzkonstruktion: 9/10 große F., 1 mäßige F.;

- Wortschatzerweiterung: 9/10 große F., 1 mäßige F.;

- Flüssigkeit im Sprechen: 9/10 große F., 1 mäßige F.;

1. Zur eigenen Rolle im Stück: Alle Befragten sind mit ihrer Rolle sehr bzw. außerordentlich zufrieden. Die Gründe hierfür sind unterschiedlich. Während die eine Hälfte der TN angibt, „die Rolle ist mir selbst sehr nah", erklärt die andere Hälfte, die Rolle sei besonders weit vom eigenen Selbst entfernt und daher besonders attraktiv für sie (s.o.). Die Mehrheit betrachtet ihre Rolle darüber hinaus als "eigene Kreation, auf die ich etwas stolz sein kann". Fast alle (8/10) sind sich vollkommen sicher, dass sie in Zukunft die im Theater gelernten sprachlichen Strukturen im Zusammenhang mit der eigenen Rolle erinnern werden. 
2. Zum Aspekt der Gruppenarbeit: Alle halten die Gruppenarbeit für anregend (4/10) bzw. sehr anregend (6/10). Fast ausnahmslos fühlten sich alle gleichberechtigt in die verschiedenen Arbeitsphasen und prozesse einbezogen. In der Listenfrage wählten sie unterschiedliche Vergleiche, um die Situation in der Gruppe zu definieren (Mannschaft, Orchester, Chor, etc.), wobei die Mehrheit den Begriff „Mannschaft“ ankreuzte.

3. Zum Text: Alle TN fühlten sich bei der Erarbeitung des Textes sowie in Regie-Entscheidungen gleichberechtigt miteinbezogen. Die Frage, ob sie lieber mit einer fertigen Textvorlage gearbeitet hätten, wurde von allen Befragten verneint. Auf die offene Frage, aus welchen Gründen sie die eigene Erarbeitung bzw. die Adaption eines Stückes vorziehen, gab es folgende Antworten (auffällig ist die Rekurrenz des Wortes eigen!):

- „Wir hätten nicht unsere eigenen kreativen Ideen im Blick auf die Aufführung umsetzen können." (Mattia S.)

- „Es war schön, sich einen bekannten Text zu eigen zu machen und ihn in die heutige Zeit zu projizieren, ohne dessen Grundprinzipien zu verletzen, d.h. die Struktur und die Themen dabei intakt zu lassen“. (Riccardo B.)

- „Ein selbst erstellter Text ist etwas, was man als sein EIGENES betrachtet, weil es etwas Originelles ist". (Elisabetta C.)

- „selbst etwas zu kreieren motiviert mehr, an den einzelnen Szenen zu feilen“. (Eliana S.)

- „es ist lustiger und aufregender, die eigenen Vorstellungen und Ideen auf die Bühne zu bringen“. (Martina B.)

- „Es gibt nichts Spannenderes, als wenn man sich frei fühlt, die eigene Rolle und den Text mit dem individuellen Zutun zu bereichern." (Alessio T.)

Insgesamt ergibt sich das Bild von TN, die sich ihrer sprachlichen Fortschritte nach Abschluss der Theaterarbeit bewusst sind und darüber hinaus die Schaffens-Freiheit, die ihnen bei der Erarbeitung der Stücke gewährleistet wurde, zu schätzen wissen.

\section{Individuelle Schaffensprozesse im Kontext des Kollektiven: Abschließender Kommentar}

Im Beitrag wurde versucht zu zeigen, welche individuellen und kollektiven Schaffensprozesse bei der Erarbeitung eines Theaterstückes ineinander greifen, 
und es wurden Arbeitsvorschläge angeführt, die in verstärktem Maße zur Förderung solcher Prozesse beitragen können.

Ein gemeinsames Produkt scheint - auch den Ergebnissen in Kapitel 4 nach große Zufriedenheit bei den einzelnen TN auszulösen, v.a. wenn sich diese des besonderen Wertes ihres individuellen Beitrags innerhalb der Gruppenarbeit bewusst sind. Diese Zufriedenheit über das Schaffen von „Eigenem“ im Kontext einer Gemeinschaft korreliert mit gesteigerter Lernmotivation und verbesserten sprachlichen Leistungen. Sie dürfte maßgeblich zum besseren Behalten sprachlicher Strukturen beitragen, zumal neue Ausdrücke und sprachliche Strukturen zusammen mit positiven Gefühlen enkodiert werden.

Die im Beitrag beschriebenen Verfahren sind selbstverständlich als Beispiele, nicht als Patentrezepte zu verstehen. Bekanntlich kann und darf Theaterarbeit nicht festgefahrenen Mustern unterliegen, sondern ist jedes Mal neu auf die spezifische Situation der Gruppe abzustimmen. Folgendes sei außerdem angemerkt: Größtmögliche Schaffensfreiheit zu garantieren bedeutet zweifellos auch, dass niemand von vornherein gezwungen werden kann, sich am Schaffensprozess zu beteiligen. Die Lust am Mitgestalten muss aus einem Gefühl von Sicherheit und aus der spontanen Reaktion auf Anreize innerhalb des Spielrahmens entspringen, aus der „sozialen Motivation“ heraus und nicht zuletzt auch aus dem Verantwortungsbewusstsein gegenüber der Gruppe, der man sich Schritt für Schritt mehr zugehörig fühlt. ${ }^{20}$

Eine rückblickende Bewertung der Arbeit seitens der TN erweist sich in jedem Fall für die Lehrperson als wichtige Hilfe, um die eigenen Vorgehensweisen kontinuierlich zu überdenken und möglichst zu verbessern.

\section{Bibliografie}

Bonnet, Andreas \& Küppers, Almut (2011): Kooperatives Lernen und Dramapädagogik. In: Küppers, Almut; Schmidt, Torben \& Walter, Maik (Hrsg.): Inszenierungen im Fremdsprachenunterricht. Braunschweig: Diesterweg / Klinkhardt, 180-191

Brecht, Bertolt (1968): Die Dreigroschenoper. Frankfurt/M: Suhrkamp

Franz, Susanne \& Hesse, Mechthild (2011): Vom Lesen eines Prosatextes bis zur dramatischen Aufführung. In: Küppers, Almut; Schmidt, Torben \& Walter, Maik (Hrsg.): Inszenierungen im Fremdsprachenunterricht. Braunschweig: Diesterweg / Klinkhardt, 104-116

Giebert, Stefanie (2012): Much ado about Business - Fachsprache im Theaterprojekt. In: Scenario, Jahrgang IV, 1, 2012, 64-89

\footnotetext{
${ }^{20}$ In einer von mir geleiteten Theatergruppe am Goethe-Institut Turin (2006) weigerte sich der einzige männliche Teilenehmer zunächst, eine Rolle zu übernehmen und erklärte: „Ich bin das Publikum.“ Im Laufe der weiteren Treffen wurde „das Publikum“ zu einer der interessantesten Figuren, die kritisch und humorvoll ins Geschehen eingriff und letztendlich maßgeblich an der Gestaltung der gemeinsamen Arbeit beteiligt war.
} 
[http://research.ucc.ie/scenario/2012/01/giebert/05/de, letzter Zugriff Dezember 2015]

Huber, Ruth (2003): Im Haus der Sprache wohnen. Wahrnehmung und Theater im Fremdsprachenunterricht. Tübingen: Niemeyer

Hoffmann, Sabine (2008): Fremdsprachenlernprozesse in der Projektarbeit. Tübingen: Narr

Holzkamp, Klaus (1983): Grundlegung der Psychologie. Frankfurt/M.: Campus

Johnson, David W.; Johnson, Roger T. \& Holubec, Edythe J. (1994): The nuts and bolts of cooperative learning. Minnesota: Interaction Book Company.

Johnson, David W. (2009): An Educational Psychology Success Story: Social Interdependence Theory and Cooperative Learning. In: Educational Researcher 38 (5), 365-379

Kleppin, Karin (2004): „Bei dem Lehrer kann man ja nichts lernen“. Zur Unterstützung von Motivation durch Sprachlernberatung. In: ZIF 9 (2). [https://zif.spz.tu-darmstadt.de/jg-09-2/beitrag/Kleppin2.htm, letzter Zugriff Dezember 2015]

Küppers, Almut; Schmidt, Torben \& Walter, Maik (Hrsg.) (2011): Inszenierungen im Fremdsprachenunterricht. Braunschweig: Diesterweg / Klinkhardt

Mummert, Ingrid (2006): Begegnungen mit „Gertrud” und „Elsa”. Mündliche und schriftliche Interpretation deutschsprachiger Literatur mit ausländischen Studierenden. Eine Studie. Baltmannsweiler: Schneider

Passon, Jenny (2011): othello@realschule. de - Eine englischsprachige Theaterinszenierung. In: Küppers, Almut; Schmidt, Torben \& Walter, Maik (Hrsg.): Inszenierungen im Fremdsprachenunterricht. Braunschweig: Diesterweg / Klinkhardt, 180-191

Poreda, Wolfgang (2011): Eigene Texte inszenieren. In: Küppers, Almut; Schmidt, Torben \& Walter, Maik (Hrsg.): Inszenierungen im Fremdsprachenunterricht. Braunschweig: Diesterweg / Klinkhardt, 131-140

Queneau, Raymond (2007): Stilübungen. (Exercices de style). Übersetzung: Ludwig Hartig \& Eugen Helmlé. Frankfurt/M.: Suhrkamp Verlag

Reinhardt, Michaela (2006): Was soll das Theater? In: Materialien Deutsch als Fremdsprache 76: Umbrüche. Regensburg: Fachverband Deutsch als Fremdsprache, 479-496

Reinhardt, Michaela (2009): Sprechen in den Wissenschaften - zunächst mit Humor. In: Hunstiger, Agnieszka; Koreik, Uwe (Hrsg.): Chance Deutsch. Schule-Studium-Arbeitswelt. 34. Jahrestagung Deutsch als Fremdsprache 2006 an der Univ. Hannover. Materialien Deutsch als Fremdsprache Bd.78. Regensburg: Universitätsverlag, 309-322

Reinhardt, Michaela (2010): Theaterarbeit im universitären Sprachunterricht. In: DaF-Werkstatt, 15-16/2010, Arezzo: Edizioni Biblioteca Aretina, 87-96 
Reinhardt, Michaela (2014): Theatertexte - Literarische Kunstwerke. Untersuchungen zu poetischer Sprache in zeitgenössischen deutschen Theatertexten. Berlin: Erich Schmidt

Riemer, Claudia (2004): Zur Relevanz qualitativer Daten in der neueren L2-Motivationsforschung. In: Börner, Wolfgang. \& Vogel, Klaus (Hrsg.): Emotion und Kognition im Fremdsprachenunterricht. Tübingen: Narr, 35-65

Schewe, Manfred Lukas (2000): DaF-Stunden dramapädagogisch gestalten wie mache ich das? In: Schlemminger, Gerald; Brysch, Thomas \& Schewe, Manfred Lukas (Hrsg.): Pädagogische Konzepte für einen ganzheitlichen DaF-Unterricht. Berlin: Cornelsen, 72-105

\section{Anhang}

\section{Fragebogen TiLLiT 2014/2015 (deutsche Übersetzung) Individuelle und kollektive Schaffensprozesse}

(Michaela Reinhardt)

\section{Zum Spracherwerb - allgemeine Aspekte}

1.1 Fühlst du dich nach der Theaterarbeit motivierter als vorher, Deutsch zu lernen?

Nein Ja, ein bisschen motivierter als vorher Vielmotivierter

1.2 Hast du das Gefühl, Fortschritte beim Deutschlernen gemacht zu haben? In Bezug auf...

die Aussprache: ich weiß nicht mäßige Fortschritte große

Fortschritte das Sprachverstehen: ich weiß nicht mäßige Fortschritte große Fortschritte

Satzkonstruktionen: ich weiß nicht mäßige Fortschritte große Fortschritte

Wortschatzerweiterung: ich weiß nicht mäßige Fortschritte große Fortschritte

Flüssigkeitbeim Sprechen:ich weißnicht mäßigeFortschritte große Fortschritte

Lust zu sprechen: ich weiß nich mäßige Fortschritte große

Fortschritte

\section{Zur eigenen Rolle}

Bist du mit der Rolle zufrieden, die du interpretiert hast?

(Name der Figur:

Nicht sehr_ einigermaßen sehr außerordentlich

zufrieden

Wenn ja, warum hat dir diese Figur gefallen? (du kannst eventuell auch mehrere Gründe ankreuzen)

- Die Figur ist mir selbst sehr nahe

- Die Figur ist von mir selbst weit entfernt und daher besonders reizvoll

- Es handelt sich um eine wichtige Figur im Stück 
- Ich habe gern die Rolle einer komischen

lächerlichen

bösen schlauen attraktiven bewundernswerten

(weiteres? ) Figur gespielt.

- Ich habe die Rolle als meine eigene Kreation empfunden, auf die ich etwas stolz sein kann

- Andere Aspekte:

Wenn nicht, aus welchen Gründen hat sie dir nicht gefallen? (du kannst eventuell auch mehrere bzw. alle Gründe ankreuzen)

Ich habe mich in dieser Rolle nicht wohl gefühlt

Mir ist es nicht gelungen, mich mit der Figur zu identifizieren

Ich fühlte mich gezwungen, diese Rolle zu interpretieren

Ich konnte bezüglich der Rolle wenige Entscheidungen selbst treffen

Es handelt sich um eine wenig wichtige Figur im Stück

Weiteres:

Glaubst du, dass du die sprachlichen Strukturen, die du beim Spielen deiner Rolle gelernt hast, auch in Zukunft in Zusammenhang mit dieser Rolle/Figur erinnern wirst?

Ich weißnicht Vielleicht Wahrscheinlich Sicher

\section{Zur Arbeit am Text und an der Aufführung}

Hast du dich bei der Erarbeitung des Textes miteinbezogen gefühlt?

Nein wenig sehrstark (wirwaren alle „Ko-Autoren”)

Hast du dich in Regie-Entscheidungen mit einbezogen gefühlt?

Nein_ wenig sehr stark (wir waren alle ein bisschen

Regisseure unseres Stückes)

Hättest du lieber mit einem bereits fertigen Text gearbeitet?

Ja_ Nein Ja und nein

Wenn ja, warum?

Wenn nicht, warum nicht?

\section{Zur Gruppenarbeit}

War die Gruppenarbeit anregend?

Nein Öfters gab es Schwierigkeiten anregend sehr anregend

Hast du Hierarchien in der Gruppe gespürt?

Nein, wirwaren allegleich berechtigt es gabTN, die dominierten

es herrschte eine recht starke Hierarchie; ich fühlte mich von den anderen wenig respektiert

Welche der folgenden Situationen erscheint dir am besten geeignet, um die Gruppenarbeit zu beschreiben? (du kannst auch mehr als eine angeben)

$$
\begin{aligned}
& \text { „Wir sitzen alle im gleichen Boot" } \\
& \text { Ein }
\end{aligned}
$$
Arbeit auf einer Baustelle

Wettstreit_eine Mannschaft Eine Gruppe mitReiseleiter

Ein Orchester, in dem jede(r) ein anders Instrument spielt Ein Chor Eine andere Situation: 